\title{
Venous thromboembolism prophylaxis in inflammatory bowel disease flare-ups
}

\author{
Osama Kaddouraha, Laith Numan' ${ }^{\text {, Sravan Jeepalyamb }}{ }^{\text {, Omar Abughanimeh }}{ }^{\text {, }}$ \\ Mouhanna Abu Ghanimeh', Khalil Abuamra \\ University of Missouri-Kansas City, Missouri; Henry Ford Health System, Detroit, Michigan, USA
}

\section{Abstract}

\section{Introduction}

Inflammatory bowel disease (IBD) is a chronic disease that affects the gastrointestinal tract and also has extraintestinal manifestations. IBD has 2 major disease entities: Crohn's disease and ulcerative colitis. The etiology of IBD is not fully understood, but is influenced by multiple factors, such as

Department of a Gastroenterology (Osama Kaddourah, Khalil Abuamr); ' Internal Medicine (Laith Numan, Sraven Jeepalyam, Omar Abughanimeh), University of Missouri-Kansas City, Missouri; ${ }^{\mathrm{c}}$ Gastroenterology, Henry Ford Health System, Detroit, Michigan (Mouhanna Abu Ghanimeh), USA

Conflict of Interest: None

Received 20 April 2019; accepted 15 July 2019; published online 31 August 2019

Correspondence to: Laith Numan, MD, 1751 NW $38^{\text {th }}$ ST Apt 203, Kansas City, MO, 64116, USA, e- mail: laith.numan90@gmail.com

DOI: https://doi.org/10.20524/aog.2019.0412 dysregulated activation of the mucosal immune response, genetics, and environmental conditions [1,2]. The epidemiology of IBD differs from region to region, but its overall incidence and prevalence are increasing [3]. IBD generally presents with symptoms and signs related to bowel inflammation and fibrosis. These include rectal bleeding, anemia, weight loss, abdominal pain, and diarrhea. IBD has many extraintestinal manifestations that can involve multiple systems in the body [4].

One of the well-studied extraintestinal manifestations of IBD is venous thromboembolism (VTE), which includes deep vein thrombosis and pulmonary embolism [5]. The higher risk of VTE in IBD patients is related to multiple factors. One major factor would be systemic inflammation, which causes a hypercoagulable state due to the activation of the coagulation cascade and platelet aggregation [6-8]. IBD patients were found to have a 2- to 3-fold risk of VTE compared to non-IBD patients, increasing up to 8-fold during an IBD flare. Moreover, because of their immobilization and active systemic inflammation, hospitalized IBD patients have a greater chance of developing VTE $[9,10]$. VTE in IBD patients is associated with higher mortality, morbidity and in- 
hospital cost compared to non-IBD patients [3]. Hospital stay and hospital charges were double in IBD patients who developed VTE compared to non-VTE IBD patients [11].

Given the higher mortality, morbidity, and cost associated with VTE in IBD patients, VTE prophylaxis is currently recommended in IBD guidelines [12,13]. Nevertheless, VTE prophylaxis is still underutilized in IBD patients, primarily because of the nature of IBD presentation with rectal bleeding [14]. A study showed that half of the IBD patients who developed VTE were not on any thrombotic prophylaxis at the time [14]. The risk of major bleeding was not found to increase in IBD patients receiving prophylaxis [15]. In this study, we analyzed the use of VTE prophylaxis in IBD patients presenting with a flare. In addition, we investigated the safety of using chemical prophylaxis in these patients and its effect on the risk of bleeding.

\section{Patients and methods}

\section{Sample selection}

We performed a retrospective chart review of all patients diagnosed with any IBD type and were admitted to the Truman Medical Center because of an IBD flare. Our study included all patients admitted for a flare-up of the disease during the period 2014-2017. Patients with IBD flare-up were identified by reviewing the discharge diagnosis on the discharge summary after the workup was concluded. Around 400 patients met our initial criteria, from a total of 7230 admissions during the study period. We generated a randomized sample of 233 patients and included all of these in our research (Fig. 1).

The patients included in the study were all adults above the age of 18 years, non-pregnant, diagnosed with IBD (ulcerative colitis, Crohn's disease, or unspecified) via a biopsy and who presented with IBD flare-up. The exclusion criteria included pregnancy and age younger than 18 years. Patients who had developed VTE in the 3 months before hospitalization, or were found to have VTE at presentation, were also excluded, as were those with a known hereditary risk of thrombosis or bleeding.

We utilized direct chart review to collect the data of our 233 patients, chosen via random sampling. We included quantitative data based on laboratory reports, physicians' notes and imaging reports. A universal datasheet was formulated to complete the variables for each patient. All data were collected from the encounter at which the patients presented with a flare of their disease. We also followed patients for up to 30 days post-discharge to include data about the outcome. No patients were excluded, given that our initial list included all those who fulfilled our inclusion criteria. All datasheets from all investigators were combined into a single sheet before analysis.

\section{Measures}

The primary objectives of our study were to determine: 1 ) the 30-day incidence of VTE (e.g., deep vein thrombosis and

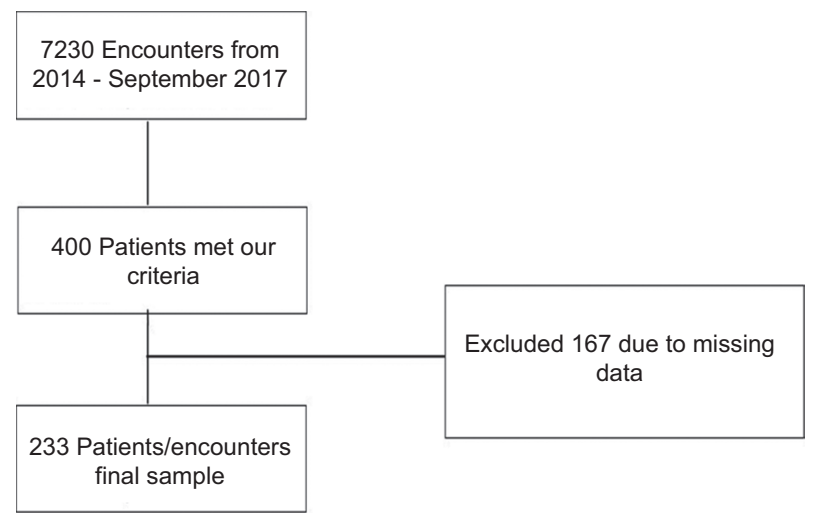

Figure 1 Study flowchart

pulmonary embolism) in hospitalized IBD patients who received chemical VTE prophylaxis versus those who received no prophylaxis; 2) the incidence of bleeding events, primarily gastrointestinal and intracranial, over 30 days postdischarge, to assess the safety of using chemical prophylaxis in IBD patients admitted for flare-up of their disease. Data from the multiple measures were collected to assess our study outcomes. These included data about the patients' demographics, comorbidities, laboratory tests, and disease description.

\section{IRB information}

IRB \#17-316: Venous Thromboembolism (VTE) Prophylaxis in Inflammatory Bowel Disease (IBD) FlareUps - A Retrospective Study. The above-referenced research was reviewed by the IRB Chair on October 18, 2017. The IRB concluded that the study is approved under the expedited Category.

\section{Statistical analysis}

Data analysis was performed using SPSS software (version 25). Normally distributed continuous variables were reported as means \pm standard deviation (SD), and categorical variables were reported as counts and percentages. Chi-square tests were used to compare categorical variables, and t-test was used to compare the continuous outcomes. We performed a multivariable logistic regression analysis for the use of VTE chemical prophylaxis. All tests were two-sided with an a level set at 0.05 for statistical significance.

\section{Results}

The 233 patients included in the study had a mean age of 36.7 (range 19-70) years. Of the patients recruited, 125 patients (53.6\%) were male and 108 (46.4\%) were female. Data about race were collected, which showed that most of our patients were 
Table 1 Demographics and clinical parameters by VTE risk factors and IBD status

\begin{tabular}{|c|c|}
\hline Characteristic & Value \\
\hline Age (years) & $36.7 \pm 10.6$ \\
\hline Male sex & $125 / 233(53.6 \%)$ \\
\hline Race & $\begin{array}{c}\text { Caucasian } 55.2 \% \\
\text { African American } 40.5 \% \\
\text { Hispanic } 3.4 \%\end{array}$ \\
\hline Body mass index $\left(\mathrm{kg} / \mathrm{m}^{2}\right)$ & $25 \pm 6.5$ \\
\hline Smoking status (present) & $116 / 233(49.8 \%)$ \\
\hline $\begin{array}{l}\text { History of venous } \\
\text { thromboembolism (present) }\end{array}$ & $5 / 233(2.1 \%)$ \\
\hline $\begin{array}{l}\text { History of gastrointestinal } \\
\text { bleeding (present) }\end{array}$ & $91 / 233(39.1 \%)$ \\
\hline $\begin{array}{l}\text { Chronic kidney } \\
\text { disease (present) }\end{array}$ & $18 / 233(7.7 \%)$ \\
\hline Intensive care unit stay & $2 / 233(0.9 \%)$ \\
\hline Malignancy (present) & Null \\
\hline $\begin{array}{l}\text { Oral contraceptive pill } \\
\text { use (present) }\end{array}$ & $8 / 233(3.4 \%)$ \\
\hline Recent surgery (present) & $1 / 233(0.4 \%)$ \\
\hline Diagnosis & $\begin{array}{c}\text { CD } 169 / 233(72.5 \%)+B 2 \\
\text { UC } 49 / 233(21 \%)\end{array}$ \\
\hline Duration disease (months) & $69 \pm 82$ \\
\hline Age of onset (A-class) & $\begin{array}{c}<16 \text { years (A1): } 21 / 169(12.4 \%) \\
17-40 \text { years }(\mathrm{A} 2): 112 / 169(66.3 \%) \\
>40 \text { years }(\mathrm{A} 3): 36 / 169(21.3 \%)\end{array}$ \\
\hline Location (L-class) & $\begin{array}{c}\text { Ileal (L1): 28/169 (16.5\%) } \\
\text { Colonic (L2): 41/169 (24.1\%) } \\
\text { Ileocolonic (L3): 97/169 (57.4\%) }\end{array}$ \\
\hline Bowel surgery (present) & $87 / 233(37.3 \%)$ \\
\hline Chronic steroids (present) & $71 / 233(30.5 \%)$ \\
\hline Hematochezia (present) & $126 / 233(54.1 \%)$ \\
\hline C-reactive protein $(\mathrm{mg} / \mathrm{L})$ & $5.6 \pm 7.2$ \\
\hline $\begin{array}{l}\text { Erythrocyte sedimentation } \\
\text { rate }(\mathrm{mm} / \mathrm{h})\end{array}$ & $35 \pm 27.7$ \\
\hline $\begin{array}{l}\text { White blood cell count } \\
\left(10^{3} / \mu \mathrm{L}\right) \text { Colonic } \\
\text { involvement }\end{array}$ & $11 \pm 5.4$ \\
\hline Colonic involvement & $\begin{array}{l}\text { Left-sided 30/49 }(61.2 \%) \\
\text { Pancolitis 17/49 }(34.6 \%)\end{array}$ \\
\hline
\end{tabular}

Caucasian (128, 55.2\% of the population), while 94 patients were African American (40.5\%), and a small proportion were Hispanic or Asian (3.4\% and $0.4 \%$, respectively). About half of our sample were smokers $(116,49.8 \%)$. The mean body mass index of the patients was 25. Further information about the patient population and the disease characteristics is shown in Table 1.

Most of our patients were diagnosed with Crohn's disease (72.5\%), while 49 patients were diagnosed with ulcerative colitis (21\%). Upon review of the presentation and biopsy results, 15 patients were deemed to have an unspecified disease (6.4\%). About $20 \%$ of our patients were on biologic therapy (i.e., adalimumab, infliximab, vedolizumab). Another 18\% were on immunomodulators (i.e., azathioprine), $2 \%$ were on a combination therapy of biologics and immunomodulators, while $40 \%$ of patients were not on maintenance therapy at the time of our study.

About a third of our patients were taking chronic steroids at the time of observation. Data regarding previous bowel surgeries related to disease were collected in approximately one third of the patients. Patients with Crohn's disease were stratified according to the region affected by the disease. Nearly half of the patients had an ileocolonic disease, whereas one-third had colonic disease only. Most of the patients with ulcerative colitis had either pan-colonic involvement or left colon involvement. One hundred twenty-six patients presented with hematochezia (54.1\%). About half of the patients had elevated C-reactive protein (laboratory value above 5) and half of the patients had leukocytosis on presentation (laboratory value above $12 \times 10^{3} / \mu \mathrm{L}$ ).

Of the patients admitted our institute with IBD flare-up, $39.7 \%$ were given chemical prophylaxis over an average duration of 4.4 days. Seventy-seven patients on chemical prophylaxis received heparin, while the rest received enoxaparin. Variables correlated with VTE prophylaxis use are included in Fig. 2. The use of chemical prophylaxis correlated significantly with male sex (odds ratio [OR] 3.4, 95\% confidence interval [CI] 3.1-3.6), recent surgery (OR 1.06, 95\%CI 1.05-1.07), history of VTE (OR 1.39, 95\%CI 1.18-1.65), current smoking (OR 1.18, 95\%CI 1.1-1.25), current chronic steroid use (OR 1.5, 95\%CI 1.471.69 ), and history of chronic kidney disease (CKD) (OR 1.24, 95\%CI 1.13-1.366). In contrast, the presence of hematochezia (OR 0.13, 95\%CI 0.122-0.14), aspirin use (OR 0.44, 95\%CI 0.21-0.89), and history of gastrointestinal bleeding (OR 0.501, 95\%CI 0.46-0.54) showed a significant negative correlation with the use of chemical prophylaxis. Patients receiving chemical prophylaxis had lower odds of overall bleeding events (OR 0.192, 95\%CI 0.141-0.263; $\mathrm{P}<0.001$ ), and there was not any significant difference in hemoglobin levels compared to patients not on chemical prophylaxis $(\mathrm{P}=0.64)$; there was no significant correlation between the hemoglobin value at presentation and the initiation of chemical VTE prophylaxis, nor any correlation with the severity of the disease.

\section{Discussion}

We recruited patients into our study retrospectively, using a database of patients admitted for IBD flare-up. The patients were relatively young, with a mean age of around 37 years old. This mean age is close to the age groups we see in the IBD clinic at our hospital, with outliers seen in the elderly, while we do not treat patients younger than 18 years of age. The average duration of the disease is around 2 years, which makes the average age of diagnosis around 35 years old in our sample. This age distribution is believed to represent the population of 


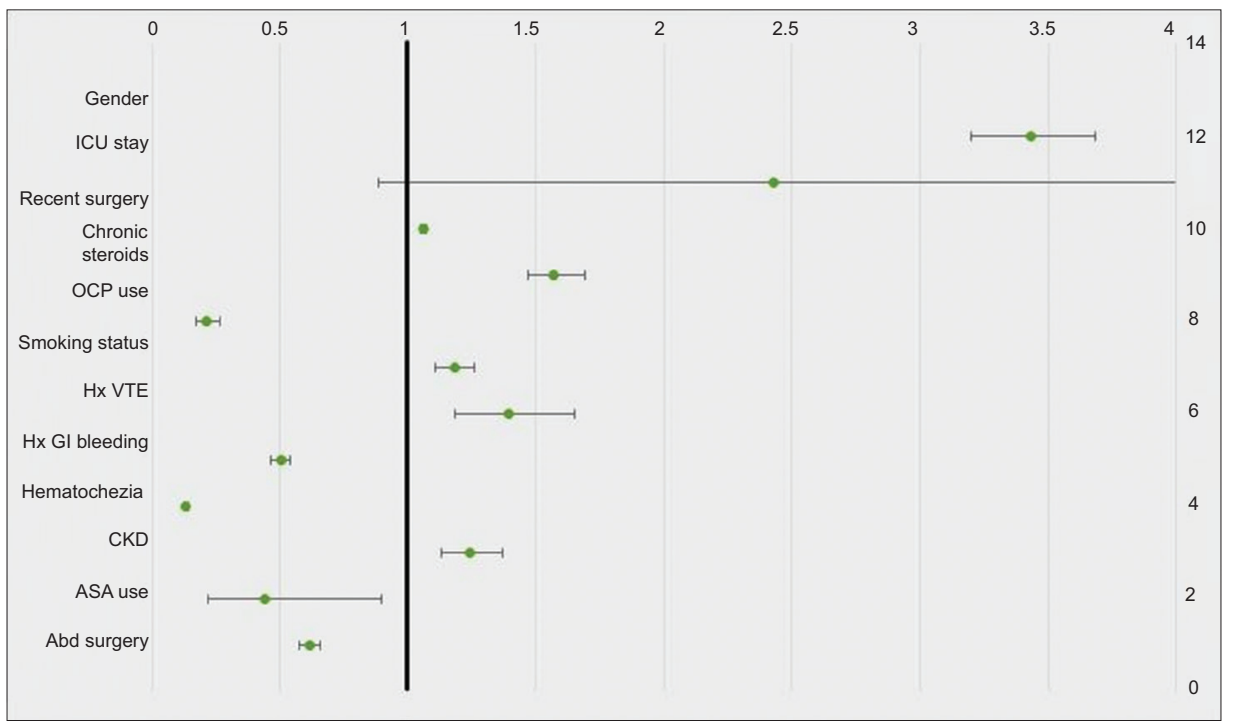

Figure 2 Variables correlated with the use of VTE prophylaxis in IBD patients

ICU, intensive care unit; OCP, oral contraceptive pill; VTE, venous thromboembolism; GI, gastrointestinal; CKD, chronic kidney disease; ASA, aspirin; Abd, abdominal

patients admitted for IBD flare-up in our hospital, given the size of the sample and randomized sampling. Although most of the patients included were Caucasian and of male sex, this predominance is not thought to be significant.

In regard to risk factors for VTE in IBD patients, smoking is a common risk and it is discouraged in patients with IBD. We routinely counsel IBD patients on tobacco use, as we believe it can increase the risk of exacerbation. Around half of our sample were smokers. The analysis of data based on smoking status showed that smokers have higher odds to receive chemical prophylaxis for VTE (OR 1.18, 95\%CI 1.1-1.25). This could be related to smoking status per se, where physicians become more concerned about the risk of VTE and initiate prophylaxis. Alternatively, it could be associated with the medical condition of patients with positive smoking status, who might have more comorbidities and more cardiac risk factors that alter the decision regarding VTE prophylaxis. Our observations do not indicate that smoking decreases the risk of hematochezia upon presentation, nor that it decreases the overall gastrointestinal bleeding risk.

Our sample consisted mainly of young patients who had frequent admissions for IBD flare-ups. This trend in age is generally seen in our institute, with the disease affecting young patients more and having equal distribution between the sexes. Given their young age, many patients have no concurrent comorbidities and are not taking multiple medications regularly. Half of our sample were smokers, which puts them at increased risk of disease progression and recurrence. Crohn's disease was dominant in our population, featuring in more than $70 \%$ of the cases. Imaging studies with evidence of extracolonic involvement and known IBD may be sufficient to diagnose Crohn's disease, which might make diagnosis more straightforward in such cases. Patients with Crohn's disease may have complications related to strictures and fistulization, which forces them to attend hospital and be admitted. This could explain, in part, why many of the patients included in our study had Crohn's disease. This distribution could have affected our external validity, with only $21 \%$ of the sample diagnosed with ulcerative colitis, especially given that ulcerative colitis is more closely associated with hematochezia and exclusively affects the colon.

One of the other risks for VTE is the chronic use of corticosteroids, which we defined as current use of various doses of corticosteroids at the time of admission for flare-up (one third of our patients). This could be related to a recent flare-up of disease or uncontrolled disease on maintenance therapy. We included those patients, since they are at increased risk for thrombotic events due to steroid use. In a metaanalysis, Sarlos et al found that the use of steroids in IBD patients was significantly associated with a higher risk of VTE when compared with IBD patients not on steroids [16]. Those findings certainly made physicians more liberal and comfortable with the use of chemical prophylaxis in such patients, given their higher risk. Our results support this, as we found a higher rate of chemical prophylaxis use in individuals on chronic steroids (OR 1.5, 95\%CI 1.47-1.69). We also found that patients with a history of VTE were more likely to receive VTE chemical prophylaxis (OR 1.39, 95\%CI 1.18-1.65). Physicians taking care of such patients probably perceived the increased risk of recurrent VTE in patients with a positive history. This was confirmed in multiple studies showing that a history of VTE is a risk factor for recurrent VTE [17]. However, we also know that IBD status independently increases the risk of VTE, and that all patients should be on VTE prophylaxis regardless of the status of previous VTE.

We also found that CKD is one of the diseases that places the patient at higher risk of developing VTE, although the stage of CKD was not specified [18]. There are no specific data concerning IBD patients with CKD. In our study, having $\mathrm{CKD}$ was associated with an increased use of VTE chemical 
prophylaxis, which can be explained by the independent increase of VTE risk in those patients (OR 1.24, 95\%CI 1.131.366). Another risk of developing VTE is having undergone recent surgery, especially high-risk orthopedic surgeries. However, even low-risk operations were associated with a small increase in VTE risk [17]. This increased risk is usually addressed by putting these patients on prophylaxis, despite their other risk factors. IBD patients are already at higher risk of VTE, elevated by recent surgery. We found that patients who had a history of recent surgery are more likely to receive chemical prophylaxis, given the higher risk mentioned above (OR 1.06, 95\%CI 1.05-1.07).

On the other hand, we found that patients on low-dose aspirin at home before the presentation are less likely to receive chemical VTE prophylaxis (OR 0.44, 95\%CI 0.217-0.893). This can be attributed to the perceived increased risk of bleeding due to the use of aspirin, which inhibits platelet activation and aggregation by inhibiting the cyclooxygenase enzyme. This, in theory, prevents clotting and can increase the risk of bleeding. Physicians might be more hesitant to initiate VTE prophylaxis in patients with such an increased risk of bleeding, confirmed in our study. There was no significant correlation between aspirin use and a drop in hemoglobin level during admission, regardless of the status of VTE prophylaxis.

Interestingly, the use of oral contraceptives in our patients made it less likely for them to receive VTE chemical prophylaxis (OR 0.212, 95\%CI 0.171-0.265). Oral contraceptive use (especially estrogen formulas) is a known risk factor for VTE [18]. Failure to provide VTE chemical prophylaxis to such patients with multiple risk factors places them at increased risk of VTE. We cannot explain such results, which could easily be confounded by other variables since we did not conduct stratified analysis of the use of oral contraceptive pills in our study. In addition, patients with a history of abdominal surgeries related to IBD were less likely to receive VTE prophylaxis, which we could not attribute directly to a specific factor. One theory would be that physicians perceive patients with a history of abdominal surgery to be "cured" of IBD, or at least to have less inflammatory burden after surgery. As mentioned above, inflammatory burden correlates with the risk of VTE. This theory, however, is at odds with the fact that our patients presented with flare-ups of their inflammation, which puts them at risk of VTE. This is regardless of any therapeutic measures, including previous surgeries performed. Curative surgeries such as colectomy (elective and emergent) for ulcerative colitis patients were also found to increase the risk of VTE after surgery, regardless of disease status [19].

Our results confirm the report by Dwyer et al of the underutilization of VTE prophylaxis in IBD patients [14]. In our institute, we found that the utilization of VTE prophylaxis was only $39.7 \%$. Other studies showed variable rates of VTE prophylaxis use in their institutes. These rates are still suboptimal, ranging from $25-80 \%$ of IBD patients admitted for various reasons $[15,20,21]$.

Most IBD patients present with bloody diarrhea at the time of the flare, which makes it challenging for the admitting physician to start them on pharmacological VTE prophylaxis, given the high risk of bleeding. However, a study by Ra et al, which assessed the safety of VTE prophylaxis in IBD patients, found that the bleeding risk with VTE prophylaxis is not higher than in its absence [15]. Our results showed that the use of VTE prophylaxis is safe, and the drop in hemoglobin level over the admission period was not different from the decline noted in patients not on prophylaxis. The underutilization of VTE prophylaxis in our institute encouraged us to follow this study with a quality improvement project to improve the rate of VTE prophylaxis.

The increased mortality and morbidity in IBD patients who develop VTE was the trigger for our study, as we were exploring the utilization of VTE prophylaxis in our institute and trying to prevent a complication that can be easily avoided by adherence to VTE prophylaxis. Nguyen et al described the detrimental effect of VTE on IBD patients' mortality and morbidity compared to non-IBD patients [10]. They found that VTE in hospitalized IBD patients leads to more than 2-fold greater mortality when compared to hospitalized non-IBD patients, with the results adjusted for age and comorbidities. Moreover, they reported an estimated $17 \%$ annual rise in VTE among IBD admissions. These findings make it clear that action should be taken as soon as possible to attempt to prevent this inclement rise of VTE in IBD patients.

Limitations of this study were that it was single-center retrospective and that the characteristics of our patient population were not matched. Also, more than $72 \%$ of our population were diagnosed with Crohn's disease. We believe that our findings might not apply to the general population, but they reflect a general trend in US hospitals for the underutilization of VTE prophylaxis for IBD patients. Multicenter prospective studies on a large sample might help confirm these findings and generalize them to all types of IBD. Unfortunately, we could not capture enough VTE events postdischarge to include in our analysis regarding our primary outcome. This might be due to the relatively short follow up period (30 days post discharge) or losing patients to follow up, given that this was a retrospective data collection.

In conclusion, our findings prove that insufficient progress has been made in terms of implementing the IBD guidelines regarding VTE prophylaxis in hospitalized IBD patients. We found multiple factors associated with the use of pharmacological prophylaxis in hospitalized patients, including sex, steroid use, history of VTE events, and gastrointestinal bleeding. In line with the results of previous studies, we found no increase in the risk of bleeding in IBD patients using chemical prophylaxis during flare-ups. Despite the documented safety of VTE prophylaxis, the risk of bleeding still plays a major role in limiting the use of VTE prophylaxis, although we have to keep in mind that a balance should be struck between the benefits and risks. Based on the findings from our study and previous research, it is clear that the benefit of preventing VTE in IBD patients outweighs the risk of bleeding from VTE prophylaxis.

\section{Acknowledgment}

We are grateful for the research staff at UMKC who helped us complete this study 


\section{Summary Box}

\section{What is already known:}

- Patients with inflammatory bowel disease (IBD) are at a higher risk of developing venous thromboembolism (VTE) due to systemic inflammation

- VTE in IBD patients is associated with higher mortality and morbidity

- VTE prophylaxis is recommended in the guidelines for IBD patients

- VTE prophylaxis is underutilized in hospitalized IBD patients admitted for various reasons

\section{What the new findings are:}

- Found variables that could affect the decision making of admitting physicians in ordering VTE prophylaxis for IBD patents.

- These variables predict a high risk for thrombotic events, including previous VTE, previous surgeries, obesity, smoking and chronic steroids. Others predict a high risk for bleeding, including anticoagulation, antiplatelet medications and a history of bleeding events

- The underutilization of VTE prophylaxis is not a new finding, but this study found that it is still an ongoing issue, despite all the published guidelines

\section{References}

1. Podolsky DK. Inflammatory bowel disease. $N$ Engl J Med 2002;347:417-429.

2. Orholm M, Munkholm P, Langholz E, Nielsen OH, Sørensen TI, Binder V. Familial occurrence of inflammatory bowel disease. N Engl J Med 1991;324:84-88.

3. Molodecky NA, Soon IS, Rabi DM, et al. Increasing incidence and prevalence of the inflammatory bowel diseases with time, based on systematic review. Gastroenterology 2012;142:46-54.

4. Olpin JD, Sjoberg BP, Stilwill SE, Jensen LE, Rezvani M, Shaaban AM. Beyond the bowel: extraintestinal manifestations of inflammatory bowel disease. Radiographics 2017;37:1135-1160.

5. Solem CA, Loftus EV, Tremaine WJ, Sandborn WJ. Venous thromboembolism in inflammatory bowel disease. Am J Gastroenterol 2004;99:97-101.
6. Saibeni S, Saladino V, Chantarangkul V, et al. Increased thrombin generation in inflammatory bowel diseases. Thromb Res 2010;125:278-282.

7. Collins CE, Cahill MR, Newland AC, Rampton DS. Platelets circulate in an activated state in inflammatory bowel disease. Gastroenterology 1994;106:840-845.

8. Gris JC, Schved JF, Raffanel C, et al. Impaired fibrinolytic capacity in patients with inflammatory bowel disease. Thromb Haemost 1990;63:472-475.

9. Wallaert JB, De Martino RR, Marsicovetere PS, et al. Venous thromboembolism after surgery for inflammatory bowel disease: are there modifiable risk factors? Data from ACS NSQIP. Dis Colon Rectum 2012;55:1138-1144.

10. Nguyen GC, Sam J. Rising prevalence of venous thromboembolism and its impact on mortality among hospitalized inflammatory bowel disease patients. Am J Gastroenterol 2008;103:2272-2280.

11. Yuhara H, Steinmaus C, Corley D, et al. Meta-analysis: the risk of venous thromboembolism in patients with inflammatory bowel disease. Aliment Pharmacol Ther 2013;37:953-962.

12. Travis SP, Stange EF, Lémann M, et al; European Crohn's and Colitis Organisation (ECCO). European evidence-based Consensus on the management of ulcerative colitis: Current management. J Crohns Colitis 2008;2:24-62.

13. Van Assche G, Dignass A, Reinisch W, et al; European Crohn's and Colitis Organisation (ECCO). The second European evidencebased Consensus on the diagnosis and management of Crohn's disease: Special situations. J Crohns Colitis 2010;4:63-101.

14. Dwyer JP, Javed A, Hair CS, Moore GT. Venous thromboembolism and underutilisation of anticoagulant thromboprophylaxis in hospitalised patients with inflammatory bowel disease. Intern Med J 2014;44:779-784.

15. Ra G, Thanabalan R, Ratneswaran S, Nguyen GC. Predictors and safety of venous thromboembolism prophylaxis among hospitalized inflammatory bowel disease patients. J Crohns Colitis 2013;7:e479-e485.

16. Sarlos P, Szemes K, Hegyi P, et al. Steroid but not biological therapy elevates the risk of venous thromboembolic events in inflammatory bowel disease: a meta-analysis. J Crohns Colitis 2018;12:489-498.

17. Heit JA. Risk factors for venous thromboembolism. Clin Chest Med 2003;24:1-12.

18. Dobrowolski C, Clark EG, Sood MM. Venous thromboembolism in chronic kidney disease: epidemiology, the role of proteinuria, CKD severity and therapeutics. J Thromb Thrombolysis 2017;43:241-247.

19. Kaplan GG, Lim A, Seow CH, et al. Colectomy is a risk factor for venous thromboembolism in ulcerative colitis. World $J$ Gastroenterol 2015;21:1251-1260.

20. Mathers B, Williams E, Bedi G, Messaris E, Tinsley A. An Electronic alert system is associated with a significant increase in pharmacologic venous thromboembolism prophylaxis rates among hospitalized inflammatory bowel disease patients. J Healthc Qual 2017;39:307-314.

21. Tinsley A, Naymagon S, Enomoto LM, Hollenbeak CS, Sands BE, Ullman TA. Rates of pharmacologic venous thromboembolism prophylaxis in hospitalized patients with active ulcerative colitis: results from a tertiary care center. JCrohns Colitis 2013;7:e635-e640. 